\title{
Numérisation et comportements humains : l'exemple de la fatigue cognitive des opérateurs aériens
}

\author{
Digitalization and human behaviors: the example of air operators'
} cognitive fatigue

\author{
Mick Salomone ${ }^{1}$, Ludovic Fabre ${ }^{2}$, Pierre Barbaroux ${ }^{2,3,4}$ \\ ${ }^{1}$ ONERA, Département Traitement de l'Information et Systèmes, Salon Air, micksalomone@gmail.com \\ ${ }^{2}$ Centre de Recherche de l'école de l'air (CReA), Ecole de l'air, Salon de Provence, ludovic.fabre@ecole-air.fr \\ ${ }^{3}$ Chaire « Cyber-résilience aérospatiale » de l'armée de l'air, Salon de Provence, pierre.barbaroux@ecole-air.fr \\ ${ }^{4}$ Université de Strasbourg, BETA, CNRS UMR 7522.
}

RÉSUMÉ. L'essor des technologies numériques a considérablement modifié notre quotidien et cette évolution est d'autant plus marquée dans le secteur aéronautique. Le développement de ces technologies au sein de ce secteur permet de répondre à plusieurs enjeux avec en priorité l'amélioration de la sécurité aérienne. Cependant, les modifications qu'elles génèrent sur l'activité de l'opérateur aérien peuvent avoir des conséquences négatives. La connaissance de l'état cognitif de l'opérateur aérien devient un élément essentiel pour la sécurité et en particulier la fatigue cognitive. Cette fatigue perturbe le fonctionnement cognitif de l'opérateur et peut engendrer des erreurs. II est donc nécessaire d'approfondir dans un premier temps nos connaissances concernant cet état notamment en déterminant ses corrélats cérébraux et, dans un second temps d'envisager le développement d'interfaces cerveau-machine pouvant détecter son apparition en temps réel. Enfin, l'étude de la fatigue cognitive met en exergue l'intérêt d'une approche interdisciplinaire entre l'ingénierie cognitive et l'ingénierie système.

ABSTRACT. The development of digital technologies significantly changes our daily lives and particularly the aeronautic sector. Digital technology enables flight safety but modifies operators' activity. Moreover, monitoring operators are essential for detecting any threat or error that can lead to negative consequences. Among them, cognitive fatigue is likely to alter operators' cognitive functioning and could lead them to make mistakes. The identification of its brain correlates is important in order to develop tools such as brain-computer interfaces. Finally, cognitive fatigue is an example that highlights the necessity to question the benefits that might emerge from a mutual sharing of knowledge between cognitive engineering and systems engineering.

MOTS-CLÉS. Aéronautique, Numérisation, Fatigue cognitive, Interface cerveau-machine, Ingénierie cognitive, Ingénierie système.

KEYWORDS. Aeronautic, Digitalization, Cognitive fatigue, Brain-computer interface, Cognitive engineering, Systems engineering.

L'industrie aéronautique connait une importante évolution à travers la numérisation de ses technologies de production de biens et de services, de contrôle de l'activité aérienne et de formation des opérateurs. La numérisation permet d'améliorer la sureté et la sécurité des activités aéronautiques mais l'erreur humaine est toujours la principale source d'accidents/incidents. L'une des conséquences de la numérisation est l'accroissement des flux et des volumes d'informations que les opérateurs doivent traiter quotidiennement. Cela génère une intensification de leur activité et augmente la vulnérabilité des systèmes et des organisations aux cyber-risques. Identifier et comprendre les facteurs qui influencent négativement l'activité cognitive ainsi que les performances des opérateurs évoluant en environnement numérisé est donc primordial.

Au cours de cet article, nous présentons les changements que la numérisation produit sur l'activité des opérateurs aériens (e.g. pilotes, contrôleurs du trafic aérien). Nous mettons en évidence le rôle de la « fatigue cognitive» sur les processus cognitifs de l'opérateur aérien et discutons des enjeux soulevés par l'étude scientifique de la phénoménologie de la fatigue cognitive pour les chercheurs, comme pour les concepteurs de systèmes aéronautiques. Nous discutons tout d'abord du concept d'interface cerveau-machine qui peut être une contremesure aux cyber-vulnérabilités induites par la numérisation (c) 2018 ISTE OpenScience - Published by ISTE Ltd. London, UK - openscience.fr 
croissante des environnements de travail. Puis, nous suggérons que le phénomène d'émergence et, éventuellement, de renforcement de la fatigue cognitive est dépendant de l'expertise des opérateurs. L'article s'achève par une discussion de l'intérêt de faire dialoguer les disciplines de l'ingénierie système et de l'ingénierie cognitive si l'on souhaite améliorer notre compréhension des défis de la numérisation pour les industries aérospatiale et de défense.

\section{La numérisation de l'aéronautique}

L'essor de la numérisation (cf. encadré 1) transforme considérablement nos sociétés et nos modes de vie. La numérisation fait suite à l'invention d'internet et du web ouvrant ainsi un nouveau monde dans l'innovation avec de nombreux avantages tels que le big data ou encore l'automatisation. Nous avons accès où que nous soyons à une quantité importante d'informations grâce à des appareils connectés (e.g. tablettes, Smartphones). Ces nouvelles technologies ont aussi révolutionné nos modes de communication à travers l'avènement des réseaux sociaux (e.g., Facebook, Twitter) mais également notre manière de travailler avec la modification de notre environnement de travail. Cette "révolution numérique" opère aussi au sein du secteur aéronautique civil et militaire. L'intégration de la technologie numérique a ainsi permis le développement des affichages tête haute et des glass-cockpits dans les aéronefs (civils et militaires). Des avions de combat, tels que le F35 américain ou le Rafale français, partagent massivement des informations et conduisent leurs missions dans des environnements réseau-centrés hyper connectés [GOD 10; BAR 11]. Le développement de l'écosystème des drones aériens (remotely piloted aircraft systems; RPAS) est également la conséquence directe de la révolution numérique affectant les capacités de communication et de navigation des aéronefs pilotés à distance. La sécurité des processus et des activités aéronautiques est aussi affectée par la numérisation. Dans l'aviation civile, l'enjeu commercial est colossal, la confiance des clients étant dépendante de la capacité des avionneurs de maintenir un taux d'accident/incident minimal. Le maintien d'un haut niveau de sécurité en matière de navigabilité et de gestion du trafic aérien est un enjeu majeur du développement de l'industrie des systèmes de drones aériens.

\section{La numérisation et le cyberespace : quelques éléments de définition}

Le mot «numérique» est un mot que nous employons et que nous rencontrons de plus en plus fréquemment sans vraiment pouvoir le spécifier clairement. C'est essentiellement grâce au développement de l'informatique et notamment du web que son emploi s'est démocratisé. Le numérique est tout d'abord un type de donnée constituant la base du fonctionnement des objets électroniques que nous manipulons aujourd'hui. Ainsi, lorsque nous utilisons le terme de numérisation, c'est parfois pour évoquer le passage des anciennes technologies vers les nouvelles manipulant ces informations chiffrées, c'est-à-dire numériques. Mais bien souvent, l'emploi du terme "numérisation» va un peu plus loin qu'un changement de technologie puisqu'il évoque l'utilisation des Nouvelles Technologies de l'Information et de la Communication (NTIC) pour une application déterminée. D'après le Centre Interarmées de Concepts, de Doctrines et d'Expérimentations (CICDE), la numérisation correspond d'ailleurs à « l'adaptation des possibilités techniques offertes par les NTIC et leur mise en œuvre coordonnée en vue d'optimiser l'efficacité globale des forces, en particulier dans le domaine de la prise de décision, de l'exécution de la manœuvre et du traitement de l'information ». Même si cette définition est centrée sur l'aspect militaire, elle convient tout à fait à la représentation du terme dans le monde civil en indiquant que l'intégration des NTIC a pour but l'optimisation des systèmes et donc de l'activité plus globalement.

Tout comme le «numérique », il existe plusieurs définitions du cyberespace. Dans le langage courant, nous utilisons ce terme de manière interchangeable avec l'Internet, c'est-à-dire le réseau 
informatique mondial par lequel transite les données numérisées et permettant la communication entre les objets faisant partie du réseau. Il peut aussi toutefois être un peu plus large qu'Internet. Le Ministère des Armées l'a défini comme étant «un domaine global constitué du réseau maillé des infrastructures des technologies de l'information (dont Internet), des réseaux de télécommunication, des systèmes informatiques, des processeurs et des mécanismes de contrôle intégrés. Il inclut l'information numérique transportée ainsi que les opérateurs de services en ligne ».

\section{Encadré 1. Définitions de la numérisation et du cyberespace}

En pratique, les technologies numériques sont intégrées dans les cockpits des aéronefs ainsi que dans les tours de contrôle du trafic aérien. Elles accroissent l'automatisation de certaines tâches auparavant gérées par l'opérateur aérien, afin de limiter l'erreur humaine [PAR 97 ; CHI 12]. Ainsi, les avions intègrent des systèmes d'alerte de trafic et d'évitement de collision avertissant le pilote de la présence d'autres avions dans son secteur [KUC 07]. Son développement a permis de «soulager» les pilotes de la surveillance aérienne leur permettant de se concentrer sur d'autres tâches. L'ergonomie des interfaces est également transformée. Le temps des cockpits proposant une jauge analogique pour chaque information est révolu. Les écrans LCD synthétisent toutes ces informations. On assiste au développement de cockpits entièrement tactiles dans le but d'aider l'opérateur (cf. figure 1). La communication entre les aéronefs et le sol (e.g. tour de contrôle) devient plus fréquente et de nature différente (auditive et visuelle). La numérisation permet, en plus des messages auditifs, la transmission de messages écrits et la mise à jour des données en temps réel. Cette connectivité accrue avec le développement des réseaux sans fil permet aux pilotes d'obtenir directement une quantité d'informations beaucoup plus importante sur leurs écrans. Elle leur donne également la possibilité d'extraire en vol des données sur l'appareil permettant d'anticiper d'éventuelles pannes.

Il existe également un enjeu économique associé à l'intégration des technologies numériques. Les pilotes peuvent suivre en temps réel l'évolution du contexte météorologique permettant d'éviter les zones à risque et ils reçoivent d'autres informations telles que la congestion des aéroports de destination. Ce type d'information permet d'optimiser l'itinéraire de l'aéronef afin d'avoir une maitrise énergétique optimale et plus globalement d'effectuer l'activité le plus efficacement et rentablement possible.
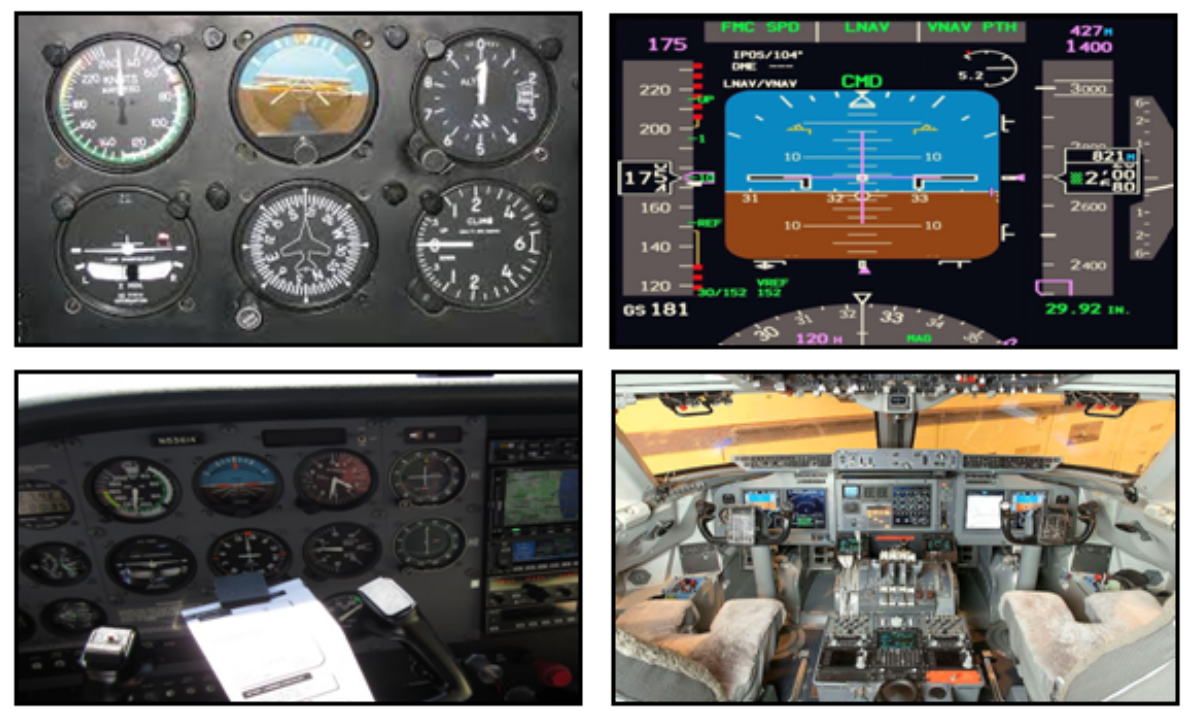

Figure 1. À gauche : un exemple de plusieurs instruments de vol analogique (haut) et la vue globale d'un cockpit incorporant ce type d'instruments (bas). À droite : l'écran primaire de navigation rassemblant plusieurs instruments analogiques (haut) et la vue globale du " glass cockpit » d'un Boeing 747 (bas). 


\section{Les effets négatifs de l'intégration des technologies numériques sur l'opérateur aérien}

L'opérateur aérien est un élément clé dans la prise de décision. Ses capacités d'adaptation permettent de maintenir l'efficience du système. Or, les changements induits par la numérisation modifient leur activité. Il est donc nécessaire de mieux comprendre l'impact de la numérisation sur les opérateurs aériens et notamment les effets négatifs issus de l'intégration de ces nouvelles technologies.

\subsection{Le risque lié à la cyber-menace}

L'intégration des technologies numériques et notamment l'augmentation de la connectivité des systèmes introduit la problématique de la protection des données qui transitent au sein du cyberespace (cf. encadré 1 pour une définition). Cette problématique est fondamentale dans le cadre militaire puisque aujourd'hui le cyberespace est considéré comme le cinquième espace de conflictualité (avec l'air, la terre, la mer et l'espace). La cyber-menace peut être de deux types: le cyber-espionnage et les cyber-attaques [voir WAN 15 pour une revue]. Le cyber-espionnage consiste à pénétrer les réseaux pour trouver un moyen de voler des informations, préparer les réseaux pour les pirater ou en prendre le contrôle. La cyber-attaque consiste à endommager (en termes de disponibilité, d'intégrité ou de confidentialité) les informations et/ou les systèmes qui les traitent, pouvant ainsi nuire aux activités dont ils sont le support (d'après le CICDE). Il peut par exemple s'agir d'une modification ou de la destruction de certaines informations mises à disposition du pilote ou du contrôleur aérien. De tels évènements peuvent bien évidemment avoir des conséquences dramatiques.

L'opérateur humain est particulièrement visé par les cyber-attaques. Dans une telle situation, son activité va fortement se complexifier et sa conscience de la situation peut être dégradée. L'état de la conscience de la situation fait référence à la sélection et au traitement des éléments de l'environnement dans lequel évolue l'opérateur (i.e., quel élément sélectionner et comment le traiter) dans le but de continuer à accomplir efficacement son activité notamment en permettant une prise de décision correcte. L'individu doit percevoir les états, les différents attributs et la dynamique des éléments de l'environnement. Il doit comprendre la situation, solliciter sa capacité de raisonnement, de décision ou encore de résolution, tout en maintenant dans la mesure du possible une certaine efficience dans son activité. Cette complexification va le soumettre à une augmentation de la charge cognitive issue de la complexification de son activité et entraine une probabilité plus importante de commettre des erreurs.

\subsection{Une augmentation considérable de la quantité d'informations à traiter}

La numérisation permet la transmission d'une quantité d'informations plus importante vers l'opérateur aérien avec l'utilisation des glass-cockpits. Ces derniers centralisent un grand nombre d'informations auparavant présentées à travers des cadrans individuels (Cf. figure 1). Cette évolution permettant de faciliter l'accès à une information peut surcharger le pilote qui rencontre alors des difficultés à traiter l'ensemble des informations ou extraire une information en particulier [CHI 12]. Un phénomène connu en aéronautique est la « fixation attentionnelle » ou « tunnel attentionnel» [WIC 09] qui peut apparaître lorsque l'opérateur focalise son attention sur une information spécifique au détriment d'autres informations pouvant parfois être vitales comme dans le cas d'une alarme [REG 14].

La numérisation peut rendre l'activité de l'opérateur aérien plus complexe et nécessiter des efforts cognitifs plus importants. La section suivante montre comment l'augmentation significative des efforts cognitifs entraine une fatigue cognitive susceptible, à certaines conditions, de favoriser l'apparition d'un état psychologique, émotionnel et/ou sensoriel dégradé. Cet état est connu pour perturber le fonctionnement cognitif humain et augmente le risque d'erreurs des opérateurs [e.g. KAM 11]. 


\section{La fatigue cognitive}

La fatigue cognitive fait partie de ces notions dont nous avons tous fait l'expérience dans la vie quotidienne [KLU 13]. C'est un type de fatigue spécifique que l'on distingue notamment de la somnolence. Deux caractéristiques de l'activité sont à l'origine de son apparition : la durée [SAN 14] et la complexité, c'est-à-dire la charge cognitive induite par sa réalisation [MAS 10]. L'utilisation de certains processus cognitifs nécessaires à l'accomplissement de tâches complexes nécessite la consommation de ressources cognitives (e.g., l'attention) disponibles en quantité limitée [KAH 73]. Si une tâche nécessite plus de ressources cognitives que ce qui est disponible, elle sera accomplie avec moins d'efficacité (cf. encadré 2 pour plus d'informations sur les ressources cognitives). Cette diminution des ressources cognitives provoquée par la réalisation d'une tâche complexe pendant une certaine durée va être associée à l'apparition de la fatigue cognitive. Son apparition peut-être très rapide (une dizaine de minutes [BOR 16]). Toutefois, la complexité et la durée de la tâche ne sont pas les seuls facteurs expliquant l'apparition de la fatigue cognitive. La quantité d'effort allouée à l'activité va être dépendante du niveau de motivation de l'individu. En effet, il est possible d'effectuer une évaluation du rapport entre l'effort lié à l'accomplissement de l'activité et les bénéfices/coûts que l'on obtient à l'accomplir. La fatigue cognitive peut apparaître lorsque cette évaluation penche davantage vers les coûts [BOK 08 ; KUR 13]. Boksem et al. (2006) ont observé que la possibilité de gagner une récompense (i.e., une motivation) permet un regain en performance après que celle-ci ait chuté en effectuant la tâche [BOK 06].

\section{Les ressources cognitives}

Les capacités de traitement de l'information de l'être humain sont limitées. Nous sommes en effet obligés de "filtrer » certaines informations afin de mieux en traiter d'autres et il nous est parfois difficile d'effectuer deux tâches en même temps sans que la performance de l'une d'elle soit diminuée. Pour rendre compte de cette limitation, le modèle de Kahneman (1973), bien qu'il ne soit pas le seul, est le plus cité. Selon ce modèle, les processus cognitifs et le traitement de l'information plus globalement nécessitent des ressources cognitives pour être effectué [KAH 73]. Ces ressources seraient disponibles au sein d'un « réservoir » unique qui serait limité en capacité. Cette limitation signifie qu'un partage de ces ressources serait obligatoirement exécuté envers les différents processus cognitifs. Notre efficience sur les tâches que l'on effectue est donc contrainte à cette limitation.

Toutefois, toutes les activités ne nécessitent pas la même quantité de ressources cognitives. Certains processus cognitif sont «automatiques» et consomment peu de ressources alors que d'autres dits « contrôlés » nécessitent leur utilisation.

\section{Encadré 2. Définition des ressources cognitives}

Les effets de la fatigue cognitive se reflètent à travers deux indicateurs : les indicateurs subjectifs (i.e. le ressenti de l'individu) et les indicateurs objectifs (i.e. la performance ou des marqueurs physiologiques). Un individu présentant une fatigue cognitive va généralement ressentir une perte d'énergie, de motivation et/ou des difficultés à se concentrer et peut présenter le besoin d'arrêter son activité pendant une période pouvant varier en fonction des individus. Ces sensations peuvent s'accompagner de difficultés à accomplir son activité entrainant une baisse des performances [BOK 06]. Kamzanova et al. (2011) ont demandé à leurs participants, au cours d'une tâche de contrôle du trafic aérien, de détecter des conflits potentiels entre deux avions pendant 40 minutes. Ils ont observé que le nombre de détections correctes diminuait au fil du temps [KAM 11]. Cette baisse des performances est corrélée à la diminution des ressources cognitives liée à la fatigue cognitive [FAB 12]. Les mécanismes d'adaptation, d'inhibition, de planification peuvent être également affectés par la 
fatigue cognitive [GIL 08; KAT 09]. Notre capacité à définir des actions futures ou à sélectionner une information pertinente est moins efficace lorsque nous sommes cognitivement fatigués [LOR 00 ; LOR 08 ; VAN 03 ; BOK 05 ; HOL 10].

Cependant, l'impact de la fatigue cognitive sur la baisse des performances est encore largement débattu dans la littérature. Certaines études montrent que les participants peuvent maintenir un niveau de performance constant tout au long de l'expérimentation bien qu'ils indiquent ressentir une fatigue cognitive [LIM 10]. Il est en effet possible de ressentir cette fatigue cognitive tout en étant capable de continuer son activité de manière efficiente. Nous ressentons généralement cette fatigue avant qu'une baisse des performances n'apparaisse puisque nous sommes capables d'augmenter nos efforts afin de maintenir un certain niveau de performance grâce à la mise en place de mécanismes de compensation neuronale [WAN 16]. Ces mécanismes se traduisent généralement par une augmentation de l'activité cérébrale d'une zone impliquée dans l'activité en cours ou alors par le recrutement d'autres zones cérébrales venant à leur tour augmenter leur niveau d'activation [BAR 13]. Mais inévitablement, la performance diminuera si la tâche perdure, c'est-à-dire lorsque nous ne serions plus capables de fournir cet effort.

\subsection{Les enjeux de la recherche}

Les effets négatifs associés à l'état de fatigue cognitive ont suscité l'intérêt des scientifiques depuis le début du siècle dernier [e.g. THO 00]. Toutefois, de nombreuses questions restent en suspens. Les principales raisons sont l'absence d'une définition consensuelle et des différences méthodologiques menant à des résultats parfois contradictoires.

\subsubsection{Les différences interindividuelles}

L'étude des différences interindividuelles concernant la fatigue cognitive est peu développée. La variabilité entre les individus a pourtant fait l'objet de nombreux travaux de recherche mais pour d'autres types de fatigue comme la somnolence [VAN 06 ; KIL 09]. Ce travail est essentiel pour deux raisons.

La première raison est que les personnes ne sont pas toutes égales face à la fatigue cognitive. Certains individus sont plus sensibles à son apparition. L'existence de ces différences indique donc que d'autres facteurs s'ajoutant à ceux déjà connus (i.e. complexité et évaluation du rapport coût/bénéfice) modulent l'apparition de la fatigue et de ses effets. Il y a en effet des facteurs stables chez l'individu qui vont influencer les mesures objectives et subjectives de la fatigue cognitive. Parmi ces facteurs, les traits de personnalité ou des variables motivationnelles ont été observées comme impactant le ressenti de la fatigue [MAT 12; EAR 15]. Desmond et Matthews (2009) ont observé que des conducteurs effectuant un long trajet ressentaient davantage les effets de la fatigue s'ils pensaient être fatigués [DES 09]. Ackerman et al. (2010) ont remarqué que certains traits de personnalité et certaines variables motivationnelles (e.g., le besoin de maitriser la tâche par exemple) avaient autant de poids sur le ressenti de la fatigue cognitive que l'activité elle-même [ACK 10]. Ces études ont cependant été réalisées dans des contextes particuliers (e.g. conduite automobile, tests verbaux ou scolaires ou encore étude en laboratoire). Certaines variables semblent avoir un poids différents en fonction du contexte [MAT 98].

La seconde raison est d'ordre méthodologique. Ne pas contrôler les différences interindividuelles lorsque l'on étudie les effets de la fatigue peut amener à de mauvaises interprétations des résultats expérimentaux [BOR 16]. Il existe en effet des différences interindividuelles en matière de capacités cognitives. La mémoire de travail par exemple, qui permet de retenir, manipuler une information et qui est impliquée dans de nombreuses activités (comme la résolution de problème), présente des différences interindividuelles en terme de capacités [ILK 10 ; BOR 16]. 
Il est donc important, notamment dans un contexte opérationnel et spécialement dans le secteur aéronautique où la sécurité est primordiale, d'identifier d'une part les individus susceptibles d'être confrontés à la fatigue cognitive, ainsi que les facteurs situationnels pouvant l'amplifier ou la maintenir d'autre part. Cela pourra permettre d'adapter l'activité en fonction du profil physio-psychologique de l'opérateur et de concevoir des environnements d'apprentissage, de formation et d'entrainement adaptés.

\subsubsection{Les différences intra-individuelles}

La fatigue cognitive est un processus dynamique qui fluctue en fonction de l'effort ou de la motivation des individus au cours d'une tâche. Il est donc important de considérer, en plus des mesures comportementales, la variabilité intra-individuelle. Cette dernière fait référence à la fluctuation de la performance chez une personne au cours d'activités [NES 02].

Certaines études ont montré que cette variabilité augmente avec l'âge [BIE 14] ou chez les individus atteints d'un trouble de l'attention [EPS 11 ; BUN 04]. Cette variabilité peut s'observer au niveau de l'activité électrique du cerveau [MCI 08 ; SLE 15]. Elle est associée à des changements au niveau de certaines structures cérébrales comme le lobe frontal [CAB 01 ; CAS 02 ; TAN 14] et est liée à des modulations dopaminergiques [HUL 02 ; BEL 05 ; BOK 08 ; LIM 12].

Peu d'études se sont intéressées à l'influence de la fatigue cognitive sur la variabilité cérébrale. La fatigue semble être reliée à une diminution de la variabilité intra-individuelle [BOK 06 ; VAN 06 ; KAT 09 ; LIU 10 ; TRA 07].

L'activité de pilotage et de contrôle du trafic aérien nécessite une attention constante sur de nombreuses informations et une réaction rapide aux évènements, en particulier en cas d'imprévu. Il est donc primordial d'étudier la relation entre la fatigue cognitive et la variabilité intra-individuelle notamment en situation réelle.

\subsubsection{Les corrélats cérébraux de la fatigue cognitive}

L'identification des corrélats cérébraux est la première étape pour le développement futur d'interfaces permettant de connaître l'état cognitif de l'opérateur et dans le cas de la fatigue cognitive de mettre en place des stratégies de compensation. Les outils utilisés par les neurosciences cognitives permettent de mieux comprendre le fonctionnement cérébral en état de fatigue cognitive.

Différentes mesures neurophysiologiques ont été utilisées pour identifier les caractéristiques de la fatigue cognitive. Parmi ces mesures, l'enregistrement de l'activité électrique cérébrale avec l'électroencéphalographie (EEG) a été la principale mesure utilisée et semble prometteuse pour la détecter [MUR 05]. Le signal électrique mesuré à travers l'EEG peut se décomposer en plusieurs bandes de fréquences (ou rythmes cérébraux) dont l'évolution dépend de l'état psychologique de l'individu. Il a été observé que certaines bandes de fréquences spécifiques, telle que la bande thêta, augmentait avec la fatigue [WAS 14]. Dasari et al. (2010) ont noté une variation des bandes alpha, thêta et bêta après avoir effectué une tâche de contrôle aérien durant 70 minutes [DAS 10]. On peut également corréler cet état de fatigue cognitive à des composantes électrophysiologiques spécifiques comme la composante $\mathrm{P} 300$ (correspondant à un pic positif apparaissant $300 \mathrm{~ms}$ après l'apparition d'un stimulus). Cette P300 reflète les processus attentionnels de l'individu et présente une réduction de son amplitude en état de fatigue cognitive [MOC 15].

La spectroscopie proche infrarouge a été utilisée pour évaluer les effets d'une activité soutenue. Cet outil consiste à étudier les changements au niveau de l'oxygénation cérébrale. L'augmentation de l'activité cérébrale induit une augmentation du débit sanguin et donc du niveau d'oxygène dans le sang. L'utilisation de cet outil est relativement récente et comporte de nombreux avantages par rapport à d'autres mesures neurophysiologiques. C'est un outil relativement portable et moins sensible aux 
interférences (e.g., les mouvements, PIP 14). Certaines études ont mis en évidence sa sensibilité à détecter un état de fatigue cognitive au niveau frontal ou encore une diminution de la connectivité intra-hémisphérique [ZHA 17].

Enfin, l'imagerie par résonnance magnétique fonctionnelle (IRMf) a été utilisée afin de prédire une éventuelle baisse de performance dans les structures cérébrales associées à l'apparition de la fatigue cognitive [LIM 10; LIM 12]. Lim et al. (2010) ont mis en relation le niveau d'activité cérébral de certaines régions (i.e. le thalamus et le cortex préfrontal) avec les performances d'une tâche suite à l'induction de fatigue. Selon eux, certaines personnes ne disposent pas suffisamment de ressources attentionnelles disponibles lorsque la complexité de la tâche augmente [LIM 10].

Les mesures subjectives (comme des échelles ou des questionnaires) ou comportementales (les temps de réponses ou la qualité du pilotage par exemple) n'indiquent finalement que la présence de la fatigue cognitive. Cela est problématique puisqu'un individu peut en effet faire des efforts afin de maintenir un certain niveau de performance surtout dans un contexte réel où les risques potentiels et la responsabilité engagée des individus peuvent les motiver à accomplir leur activité. Ainsi, étudier les effets de la fatigue mentale en mobilisant uniquement des mesures subjectives ou les mesures habituelles de performance peut limiter notre compréhension de la fatigue cognitive tandis que les mesures neurophysiologiques permettraient d'observer en temps réel voire de prédire l'apparition de cette fatigue cognitive même en l'absence d'effets sur les autres mesures [DAS 13].

\section{Les implications futures}

Les opérateurs aériens, de par leurs activités et l'évolution technologique, sont particulièrement exposés à la fatigue cognitive. Ils doivent par exemple effectuer plusieurs tâches simultanées telles que communiquer avec les contrôleurs aériens et piloter [MOR 06]. La gestion des informations est primordiale pour les pilotes afin d'avoir une bonne conscience de la situation [THA 91] et cela dépend des ressources cognitives des opérateurs. De plus, la complexité de leur activité varie au cours du temps (e.g., le nombre d'avions et de situations conflictuelles changent constamment) justifiant un effort cognitif important (e.g., attention, concentration) afin d'anticiper les changements et de maintenir un niveau de performance optimal [MOR 03].

Il est donc nécessaire de trouver des solutions limitant ou compensant les effets de la fatigue cognitive. L'identification des corrélats cérébraux de la fatigue cognitive peut être une première étape dans le développement d'interfaces intelligentes cerveau-machine permettant la surveillance et la modification en temps réel de l'état de l'opérateur. La formation avec des méthodes d'entrainement spécifiques liées à l'expertise ou l'adaptation de l'activité de l'opérateur ouvrent également des pistes de recherche permettant de réduire les risques liés à l'apparition de la fatigue cognitive.

\subsection{Compenser la fatigue cognitive : le développement des interfaces cerveau-machine}

Détecter en temps réel l'apparition d'états dégradés tels que la fatigue cognitive est un véritable enjeu pour la sécurité aérienne. Les interfaces cerveau-machine (ICM) constituent un outil envisageable pour accomplir la surveillance de l'état de l'opérateur. Ces ICM permettent une communication directe du cerveau vers la machine en utilisant les données obtenues par l'enregistrement électrophysiologique, l'EEG est le plus utilisé, comme une information à part entière intégrée dans l'agent artificiel [WOL 02]. Les ICM ont été développées la première fois dans les années 70 [VID 73] et ont surtout connu une application médicale, en permettant par exemple le contrôle d'une prothèse ou encore d'un ordinateur permettant aux personnes tétraplégiques de communiquer [MUL 08; WIL 09]. Cette interface permettra, en dehors d'une application dans le domaine de la santé, de renforcer l'Interaction Homme-Machine (IHM), en rendant la machine plus « intelligente », c'est-à-dire capable de s'adapter à l'opérateur. Il existe plusieurs types d'ICM, les ICM actives, réactives et passives. Chacune permettant des applications différentes [ZAN 08]. 
- Les ICM actives nécessitent la modulation volontaire de l'activité cérébrale afin de contrôler un effecteur par exemple. Il faut penser à l'activité désirée (e.g., un mouvement) pour le réaliser. En imaginant simplement le mouvement, ce type d'outil permet à une prothèse de reproduire le mouvement.

- Les ICM réactives permettent aussi le contrôle d'une application mais ici l'activité cérébrale est modulée involontairement par un événement externe (e.g., un flash de lumière). C'est parfois ce type d'ICM qui est utilisé pour permettre à des personnes paralysées de communiquer (e.g., P300 speller, FAR 88). Dans ce type d'utilisation, une personne voit un ensemble de lettres sur un écran et en sélectionne une par le regard, ce qui lui permet de pouvoir construire des phrases.

- Enfin, les ICM passives dans lesquelles l'activité cérébrale est enregistrée sans une implication active de l'individu. Elle permet de déduire des états mentaux spécifiques tels que les états émotionnelles [HER 07], le niveau de charge mentale [BLA 10], la somnolence ou encore la fatigue cognitive [voir BOR 12 pour une revue].

On peut donc imaginer qu'une fois l'opérateur fatigué, des stratégies soient mises en place telles que la réduction du nombre d'informations, la reprise de l'activité par un processus automatisé ou par un autre opérateur, le but étant de réduire le niveau de fatigue cognitive ressenti par l'opérateur. En plus d'adapter la quantité d'informations, il est possible de la présenter sous une autre modalité sensorielle. La recherche a montré que selon la modalité de présentation de l'information, celle-ci n'est pas traitée par la même zone cérébrale [WIC 08]. Un autre intérêt de ce type de mesure est de permettre la surveillance de l'état physiologique de l'opérateur sans que celui-ci ait besoin d'effectuer une quelconque action. Ce type de dispositif est à la fois moins invasif et moins contraignant pour l'opérateur, si on le compare avec d'autres dispositifs de mesure (e.g., complétion fréquente d'un questionnaire). Il est toutefois primordial que les indicateurs cérébraux de l'état recherché soient précis et fiables. Bien souvent, les études utilisent un protocole d'une durée importante pour induire la fatigue cognitive, sans prendre en compte l'aspect motivationnel ou encore la modification du niveau d'éveil de la personne. Il est donc crucial de déterminer les marqueurs cérébraux en tenant compte de ces facteurs mais aussi en utilisant des tâches se rapprochant le plus de l'activité des opérateurs aériens. Une mauvaise caractérisation de la fatigue cognitive peut générer la mise en place d'une aide alors qu'elle n'est pas (encore) nécessaire, ce qui peut produire des effets inverses de ceux escomptés [PAR 97]. La généralisation des résultats obtenus au cours de tâches menées en laboratoire dans des contextes plus écologiques est également essentielle. Toutefois, les ICM sont très sensibles à certaines variations telles que le mouvement du pilote ou la lumière ambiante. Leur développement en dehors du laboratoire impose des contraintes que les chercheurs doivent maitriser.

\subsection{L'expertise : un moyen de limiter la fatigue cognitive}

L'expertise s'accompagne de changements cognitifs qui améliore la capacité d'adaptation et le niveau de performance de l'individu (cf. encadré 3 pour une définition de l'expertise). Parce qu'il automatise certains processus dans son activité, l'expert est capable de réduire sa consommation de ressources cognitives et d'effectuer d'autres tâches en parallèle [SHI 77]. Plusieurs études ont notamment montré que les opérateurs aériens s'adaptent à la complexité de leur tâche et sont résilients dans leur activité en mobilisant des stratégies différentes (e.g., priorisation de certaines tâches) selon leur niveau d'expertise [SPE 78 ; LOF 07 ; KON 13]. Les changements induits par l'expertise ne sont pas uniquement comportementaux : l'expertise est également associée à des modifications cérébrales. Des études rapportent ainsi le développement d'une « efficience neuronale » (ou « neural efficiency») associée à l'expertise [GRA 06]. Cette efficience neuronale se caractérise par une diminution de l'activité cérébrale [ADA 14]. Ces différentes études ont mis en évidence des distinctions comportementales et cérébrales, notamment au niveau des habilités cognitives entre les experts et les novices. Les stratégies développées et l'automatisation des processus associés à l'augmentation du niveau d'expertise permettraient d'être moins sensible à l'apparition de la fatigue cognitive. Il est donc 
important de mieux connaitre la relation entre expertise et fatigue cognitive afin de mieux la contrôler. Approfondir la connaissance de la gestion de la fatigue cognitive par des experts peut apporter des indications sur la manière de guider l'apprentissage des novices ou des opérateurs qui effectuent la transition entre un environnement de travail analogique et numérique.

\section{La notion d'expert}

Un expert est un individu dont la performance démontre un certain niveau de compétence associé à un moindre niveau d'effort et qui est capable de traiter efficacement certains types de cas rares ou «difficiles». L'expert possède des compétences ou des connaissances spécialisées (i.e. spécifiques à un domaine) dérivées d'une vaste expérience de sous-domaines. Il est apprécié par ses pairs et ses jugements sont précis et fiables (définition adaptée de HOF 96).

\section{Encadré 3. La notion d'expert}

\subsection{Les apports de l'intégration des disciplines de l'ingénierie système et cognitive}

L'interaction de l'ingénierie système avec l'ingénierie cognitive répond à un double objectif. Le premier est de pouvoir proposer des systèmes adaptés aux processus et aux limites cognitives humaines dès les phases de conception du système. Il est possible de déceler des effets négatifs qui n'ont pas été pris en compte lors de la conception. Or, une détection tardive ne permet pas toujours la mise en place de correction. Le second est le bénéfice réciproque que les disciplines peuvent tirer des outils développés via une intégration de l'ingénierie système et de l'ingénierie cognitive. Les interfaces cerveau-machine (ICM) constituent un exemple d'outil intéressant permettant une symbiose entre l'agent humain et l'agent artificiel. L'ICM passive est un outil prometteur pour détecter les états mentaux des opérateurs et limiter leur apparition. Les ICM actives et réactives sont des pistes de recherche pour optimiser l'interaction Homme-machine en donnant la possibilité à l'opérateur d'interagir avec le système par le biais de son activité cérébrale et donc avec une rapidité accrue et un moindre effort.

À l'inverse, les problématiques de l'ingénierie système contribuent à approfondir nos connaissances sur l'être humain. Etant à l'initiative de développements technologiques, elle apporte des questionnements dans le domaine de l'ingénierie cognitive. La numérisation en est la preuve avec le questionnement de ses effets sur l'être humain. Elle impose à l'ingénierie cognitive de développer ses outils afin d'être adaptés aux systèmes. Les ICM qui se basent sur des mesures sensibles aux variations (e.g. mouvements) nécessitent des développements adaptés à un environnement complexe.

Ce travail interdisciplinaire semble nécessaire pour les recherches futures. Bien évidemment, des ajustements sont à effectuer avec la nécessité de mettre en avant ce que chacun peut apporter en termes de connaissances mais aussi une formalisation adaptée à chaque discipline [MAD 11]. Ce n'est que par la mutualisation des connaissances qu'une collaboration intelligente entre la machine et l'opérateur pourra être obtenue.

\section{Conclusion}

La numérisation a profondément modifié nos activités, en particulier dans le secteur aéronautique. Les développements et l'intégration des nouvelles technologies numériques permettent de répondre à certains enjeux (sureté, efficacité économique) dans le monde civil et militaire mais génèrent aussi des contraintes (e.g., la vulnérabilité aux cyber-attaques) dont certaines sont liées aux limites cognitives de l'agent humain. Cette contribution a ainsi mis en évidence le rôle de la fatigue cognitive induite par la numérisation des espaces de travail. La fatigue cognitive perturbe en effet le fonctionnement cognitif de l'opérateur qui peut avoir des difficultés à s'adapter à des situations complexes et imprévues. L'un 
des principaux enjeux de la recherche expérimentale est d'identifier l'impact de la fatigue cognitive sur l'activité des opérateurs et de déterminer les corrélats cérébraux de cette fatigue. L'identification de marqueurs cérébraux est une piste de recherche prometteuse pour améliorer les performances des ICM passives. L'amélioration de l'interaction entre l'Homme et la machine n'est pas la seule voie pour diminuer l'apparition de la fatigue cognitive. La formation est aussi cruciale puisqu'un expert dans son activité, par des modifications comportementales et cérébrales, est moins soumis à ce type de fatigue. Plus fondamentalement, ces deux voies de recherche suggèrent tout l'intérêt de favoriser les échanges entre les disciplines de l'ingénierie système et de l'ingénierie cognitive, avec pour objectif d'améliorer la conception des systèmes aéronautiques et notre connaissance des processus cognitifs humains en environnement numérisés.

\section{Bibliographie}

[ACK 10] ACKerman P. L., Kanfer R., Shapiro S. W., NeWton S., \& BeiER M. E., "Cognitive fatigue during testing: An examination of trait, time-on-task, and strategy influences”, Human Performance, 23(5), p. 381-402, 2010.

[ADA 14] Adamson M. M., Taylor J. L., Heraldez D., Khorasani A., NodA A., Hernandez B., \& Yesavage J. A., "Higher landing accuracy in expert pilots is associated with lower activity in the caudate nucleus". PloS one, 9(11), 2014.

[BAR 11] BARBAROUX P.,"Technologie polyfonctionnelle et compétences des acteurs : le cas des pilotes de chasse de l'armée de l'air", Revue Française de Gestion, 2011/3, N³, p. 29-43, 2011.

[BAR 13] BARULli D., \& STERN Y., "Efficiency, capacity, compensation, maintenance, plasticity: emerging concepts in cognitive reserve", Trends in cognitive sciences, 17(10), p.502-509, 2013.

[BEL 05] Bellgrove M. A., Hawi Z., Kirley A., Gill M., \& Robertson I. H., "Dissecting the attention deficit hyperactivity disorder (ADHD) phenotype: sustained attention, response variability and spatial attentional asymmetries in relation to dopamine transporter (DAT1) genotype", Neuropsychologia, 43(13), p.1847-1857, 2005.

[BIE 14] BielaK A. A., Cherbuin N., BunCE D., \& Anstey K. J., "Intraindividual variability is a fundamental phenomenon of aging: Evidence from an 8-year longitudinal study across young, middle, and older adulthood" Developmental psychology, 50(1), p. 143, 2014.

[Bla 10] Blankertz B., Tangermann M., Vidaurre C., Fazli S., Sannelli C., Haufe S., ... \& Müller K. R., "The Berlin brain-computer interface: non-medical uses of BCI technology", Frontiers in neuroscience, 4, 2010.

[BOK 05] BOKSEM M. A., MEIJMAN T. F., \& LORIST M. M., "Effects of mental fatigue on attention: an ERP study", Cognitive brain research, 25(1), p. 107-116, 2005.

[BOK 06] BoKsem M. A., MeIJMAn T. F., \& LORIST M. M., "Mental fatigue, motivation and action monitoring", Biological psychology, 72(2), p. 123-132, 2006.

[BOK 08] BOKSEM M.S. and TOPS, M., "Mental fatigue: costs and benefits", Brain Research Reviews, 59(1), p. 125-39, 2008.

[BOR 12] Borghini G., Astolfi L., Vecchiato G., MAtTia D., \& BABILONi F., "Measuring neurophysiological signals in aircraft pilots and car drivers for the assessment of mental workload, fatigue and drowsiness", Neuroscience \& Biobehavioral Reviews, 44, p. 58-75, 2012.

[BOR 16] Borragán G., Slama H., DestrebecQz A., \& PeigneuX P., "Cognitive fatigue facilitates procedural sequence learning", Frontiers in human neuroscience, 10, 2016.

[BUN 04] BUnCE D., MACDONALD S. W., \& HUltsch D. F., "Inconsistency in serial choice decision and motor reaction times dissociate in younger and older adults", Brain and Cognition, 56(3), p. 320-327, 2004.

[CAB 01] CABEZA R., "Functional neuroimaging of cognitive aging", dans R. CABEZA \& A. KingSTONE (dir.), Handbook of functional neuroimaging of cognition, MIT Press, Cambridge, 2001.

[CAS 02] CASTELlANOS F. X., \& TANNOCK R., "Neuroscience of attention-deficit/hyperactivity disorder: the search for endophenotypes", Nature Reviews Neuroscience, 3(8), p. 617-628, 2002.

[CHA 10] CHAmponNoIS, S., «L'adaptation de l'armée de l'air française à l'aviation à réaction (1945-1950) », Guerres mondiales et conflits contemporains, 238(2), p. 81-96, 2010.

[CHI 12] ChIAlastri A., “Automation in Aviation”, dans K. FloRIAN (dir.), Automation, InTech, Rijeka, 2012. 
[DAS 10] DASARI D., CROWE C., LING C., ZHU M., \& DING L., "EEG pattern analysis for physiological indicators of mental fatigue in simulated air traffic control tasks", dans Proceedings of the human factors and ergonomics society annual meeting, Sage Publications, Los Angeles, 2010.

[DAS 13] DASARI D., SHOU G., \& DING L., "Investigation of independent components based EEG metrics for mental fatigue in simulated ATC task", dans 2013 6th International IEEE/EMBS Conference on Neural Engineering (NER), IEEE, San Diego, 2013.

[DES 09] DeSMOND P. A., \& MATTHEws G., "Individual differences in stress and fatigue in two field studies of driving", Transportation Research Part F: Traffic Psychology and Behaviour, 12(4), p. 265-276, 2009.

[DOR 07] DORRIAN J., ROACH G. D., FletcheR A., \& DAWSON D., "Simulated train driving: Fatigue, self-awareness and cognitive disengagement”, Applied ergonomics, 38(2), p. 155-166, 2007.

[EAR 15] EARle F., Hockey B., EARle K., \& ClOUgh P., "Separating the effects of task load and task motivation on the effort-fatigue relationship", Motivation and Emotion, 39(4), p. 467-476, 2015.

[EPS 11] Epstein J. N., Langberg J. M., Rosen P. J., Graham A., Narad M. E., Antonini T. N., ... \& Altaye M., "Evidence for higher reaction time variability for children with ADHD on a range of cognitive tasks including reward and event rate manipulations", Neuropsychology, 25(4), p. 427, 2011.

[FAB 12] FABER L. G., MAURITS N. M., \& LORIST M. M., "Mental fatigue affects visual selective attention", PloS one, 7(10), 2012.

[FAR 88] FARWELL L. A., \& DONCHIN E., "Talking off the top of your head: toward a mental prosthesis utilizing eventrelated brain potentials", Electroencephalography and clinical Neurophysiology, 70(6), p. 510-523, 1988.

[GER 15] Gergelyfi M., JACOB B., OlivieR E., \& ZÉNON A., "Dissociation between mental fatigue and motivational state during prolonged mental activity", Frontiers in behavioral neuroscience, 9, 2015.

[GIL 08] Gilbert S. J., \& BuRgess P. W., "Executive function”, Current Biology, 18(3), p. 110-114, 2008.

[GOD 10] GODE, C., BARBAROUX, P., "La fabrique des usages technologiques en environnement volatil" ? Management \& Avenir, 2010/2, n³2, p.71-90, 2010.

[GRA 06] Grabner R. H., Neubauer A. C., \& Stern E., "Superior performance and neural efficiency: The impact of intelligence and expertise", Brain research bulletin, 69(4), p. 422-439, 2006.

[HER 07] HERAZ A., \& FRASSON C., "Predicting the three major dimensions of the learner's emotions from brainwaves", International Journal of Computer Science, 2(3), p. 187-193, 2007.

[HOF 96] HOFFMAN R.R., "How can expertise be defined? Implications of research from cognitive psychology", dans R. WILlIAMS, W. FAULKNER, \& J. FLECK (dir.), Exploring expertise, University of Edinburgh Press, Edinburgh, 1996.

[HOL 10] Holtzer R., Shuman M., Mahoney J. R., Lipton, R., \& Verghese, J., "Cognitive fatigue defined in the context of attention networks", Aging, Neuropsychology, and Cognition, 18(1), p. 108-128, 2010.

[HUL 02] Hultsch D. F., MacDonald S. W., \& DiXON R. A., "Variability in reaction time performance of younger and older adults", The Journals of Gerontology Series B: Psychological Sciences and Social Sciences, 57(2), p. 101$115,2002$.

[ILK 10] ILKOWSKA M., \& ENGLE R. W., "Trait and state differences in working memory capacity", dans A. Gruszka, G. Matthews, et B. Szymura (dir.), Handbook of individual differences in cognition, Springer, New York, 2010.

[KAH 73] Kahneman D., Attention and effort, Prentice-Hall, Englewood Cliffs, 1973.

[KAM 11] Kamzanova A. T., Matthews G., Kustubayeva A. M., \& JaKupov S. M., "EEG indices to time-on-task effects and to a workload manipulation (cueing)", World Academy of Science, Engineering and Technology, 80, p. 1922, 2011.

[KAT 09] KATO Y., ENDO H., \& KizUKA T., "Mental fatigue and impaired response processes: event-related brain potentials in a Go/NoGo task", International Journal of Psychophysiology, 72(2), p. 204-211, 2009.

[KIL 09] Killgore W. D., Grugle N. L., Reichardt R. M., Killgore D. B., \& Balkin T. J., "Executive functions and the ability to sustain vigilance during sleep loss", Aviation, Space, and Environmental Medicine, 80(2), p. 81-87, 2009.

[KON 13] KontOGiannis T., \& MALAKIS S., "Strategies in coping with complexity: Development of a behavioural marker system for air traffic controllers", Safety science, 57, p. 27-34, 2013. 
[KLU 13] Kluger B. M., KRuPP L. B., \& ENOKA R. M., "Fatigue and fatigability in neurologic illnesses Proposal for a unified taxonomy", Neurology, 80(4), p. 409-416, 2013.

[KUC 07] KUCHAR J. E., \& DRUMM A. C., "The traffic alert and collision avoidance system", Lincoln Laboratory Journal, 16(2), p. 277, 2007.

[KUR 13] Kurzban R., Duckworth A., Kable J. W., \& Myers J., "An opportunity cost model of subjective effort and task performance", Behavioral and Brain Sciences, 36(6), p. 661-679, 2013.

[LIM 10] Lim J., Wu W. C., Wang J., Detre J. A., Dinges D. F., \& RaO H., "Imaging brain fatigue from sustained mental workload: an ASL perfusion study of the time-on-task effect”, Neuroimage, 49(4), p. 3426-3435, 2010.

[Lim 12] Lim J., Ebstein R., Tse C. Y., Monakhov M., San Lai P., Dinges D. F., \& KwoK K., "Dopaminergic polymorphisms associated with time-on-task declines and fatigue in the Psychomotor Vigilance Test", PloS one, 7(3), 2012.

[LIU 10] LiU J., ZHANG C., \& ZHENG C., "EEG-based estimation of mental fatigue by using KPCA-HMM and complexity parameters", Biomedical Signal Processing and Control, 5(2), p. 124-130, 2010.

[LOF 07] LOFT S., SANDERSON P., NeAl A., \& MoOiJ M., "Modeling and predicting mental workload in en route air traffic control: Critical review and broader implications", Human Factors, 49(3), p. 376-399, 2007.

[LOR 08] LORIST M. M., "Impact of top-down control during mental fatigue", Brain research, 1232, p. 113-123, 2008.

[LOR 00] Lorist M. M., Klein M., Nieuwenhuis S., De Jong R., Mulder G., \& Meijman T. F., "Mental fatigue and task control: planning and preparation", Psychophysiology, 37(5), p. 614-625, 2000.

[MAD 11] MADNI A. M., "Integrating humans with and within complex systems", CrossTalk, 5, 2011.

[MAS 10] Massar S. A., Wester A. E., VolKerts E. R., \& KenEMAnS J. L., "Manipulation specific effects of mental fatigue: evidence from novelty processing and simulated driving", Psychophysiology, 47(6), p. 1119-1126, 2010.

[MAT 98] MatThews G., \& Desmond P. A., "Personality and multiple dimensions of task-induced fatigue: a study of simulated driving", Personality and Individual Differences, 25(3), p. 443-458, 1998.

[MAT 12] Matthews G., Desmond P. A., \& Hitchcock E. M., "Dimensional models of fatigue", dans G. MATthews, P. A. DeSMOND, C. NEUBAUER et P. A. HANCOCK, Handbook of operator fatigue, CRC Press, 2012.

[MCI 08] MCIntosh A. R., KovaceVIC N., \& ITIER R. J., "Increased brain signal variability accompanies lower behavioral variability in development”, PLoS computational biology, 4(7), 2008.

[MOC 15] MÖckel T., Beste C., \& WASChER E., "The effects of time on task in response selection-an erp study of mental fatigue", Scientific reports, 5, 2015.

[MOR 03] MORINEAU T., HOC J. M., \& DENECKER P., "Cognitive control levels in air traffic radar controller activity", The International Journal of Aviation Psychology, 13(2), p. 107-130, 2003.

[MOR 06] MORRIS C. H., \& LEUNG Y. K., "Pilot mental workload: how well do pilots really perform?", Ergonomics, 49(15), p. 1581-1596, 2006.

[MUL 08] MUller-PUTZ G. R., \& PfurTSCheller G., "Control of an electrical prosthesis with an SSVEP-based BCI", IEEE Transactions on Biomedical Engineering, 55(1), p. 361-364, 2008.

[MUR 05] MURATA A., UETAKE A., \& TAKASAWA Y., "Evaluation of mental fatigue using feature parameter extracted from event-related potential", International Journal of Industrial Ergonomics, 35(8), p. 761-770, 2005.

[NES 02] NeSSELROADE J. R., "Elaborating the differential in differential psychology", Multivariate Behavioral Research, 37(4), p. 543-561, 2002.

[PAR 97] PARASURAMAN R., \& RILEY V., "Humans and automation: Use, misuse, disuse, abuse", Human factors, 39(2), p. 230-253, 1997.

[PIP 14] PiPer S. K., Krueger A., Koch S. P., Mehnert J., Habermehl C., Steinbrink J., ... \& Schmitz C. H., “A wearable multi-channel fNIRS system for brain imaging in freely moving subjects", Neuroimage, 85, p. 64-71, 2014.

[REG 14] RéGis N., Dehais F., Rachelson E., Thooris C., Pizziol S., Causse M., \& Tessier C., « Formal detection of attentional tunneling in human operator-automation interactions", IEEE Transactions on Human-Machine Systems, 44(3), p. 326-336, 2014.

[SAN 14] SANDRY J., Genova H. M., Dobryakova E., Deluca J., \& Wylie G., "Subjective cognitive fatigue in multiple sclerosis depends on task length", Frontiers in neurology, 5, 2014. 
[SHI 77] SHIFFRIN R. M., \& SCHNEIDER W., "Controlled and automatic human information processing: II. Perceptual learning, automatic attending and a general theory", Psychological review, 84(2), p. 127, 1977.

[Sle 15] Sleimen-Malkoun R., Perdikis D., Müller V., Blanc J. L., Huys R., Temprado J. J., \& Jirsa V. K., "Brain dynamics of aging: multiscale variability of EEG signals at rest and during an auditory oddball task", Eneuro, 2(3), 2015.

[SPE 78] SPERANDIO J. C., "The regulation of working methods as a function of work-load among air traffic controllers", Ergonomics, 21(3), p. 195-202, 1978.

[TAN 14] TANAKA M., ISHII A., \& WATANABE Y., "Neural effects of mental fatigue caused by continuous attention load: a magnetoencephalography study", Brain research, 1561, p. 60-66, 2014.

[THA 91] THACKRAY R. I., \& TOUCHSTONE R., "Effects of monitoring under high and low taskload on detection of flashing and coloured radar targets", Ergonomics, 34(8), p. 1065-1081, 1991.

[THO 00] Thorndike E., "Mental fatigue", Psychological Review, 7(6), p. 547, 1900.

[TRA 07] Tran Y., Thuraisingham R. A., WiJesuriya N., NGUYen H. T. \& CRAig A., "Detecting neural changes during stress and fatigue effectively a comparison of spectral analysis and sample entropy", Proceedings of the 3rd International IEEE/EMBS Conference on Neural Engineering, IEEE, Kohala Coast, 2007.

[VAN 03] VAN DER Linden D., Frese M., \& MeIJMAn T. F., "Mental fatigue and the control of cognitive processes: effects on perseveration and planning", Acta Psychologica, 113(1), p. 45-65, 2003.

[VAN 06] VAn Dongen H. P., CAldwell J. A., \& CALDwell J. L., "Investigating systematic individual differences in sleep-deprived performance on a high-fidelity flight simulator", Behavior research methods, 38(2), p. 333-343, 2006.

[VID 73] VIDAL J. J., "Toward direct brain-computer communication”, Annual review of Biophysics and Bioengineering, 2(1), p. 157-180, 1973.

[WAN 15] Wang L., \& AleXander C. A., "Big Data in Distributed Analytics, Cybersecurity, Cyber Warfare and Digital Forensics", Digital Technologies, 1(1), p. 22-27, 2015.

[WAN 16] Wang C., Trongnetrpunya A., Samuel I. B. H., Ding M., \& Kluger B. M., "Compensatory neural activity in response to cognitive fatigue", Journal of neuroscience, 36(14), p. 3919-3924, 2016.

[WAS 14] Wascher E., RAsch B., SÄnger J., Hoffmann S., Schneider D., RinkenAuer G., ... \& Gutberlet I., "Frontal theta activity reflects distinct aspects of mental fatigue", Biological psychology, 96, p. 57-65, 2014.

[WIC 08] Wickens, C. D., "Multiple Resources and Mental Workload. Human Factors”, 50(3), p. 449-455, 2008.

[WIC 09] WiCKENS C. D., \& AlEXANDER A. L., Attentional tunneling and task management in synthetic vision displays. The International Journal of Aviation Psychology, 19(2), p. 182-199, 2009.

[Wil 09] Williamson J., Murray-Smith R., Blankertz B., Krauledat M., \& MÜller K. R., "Designing for uncertain, asymmetric control: Interaction design for brain-computer interfaces", International Journal of HumanComputer Studies, 67(10), p. 827-841, 2009.

[WOL 02] Wolpaw J. R., Birbaumer N., McFarland D. J., Pfurtscheller G., \& Vaughan T. M., "Braincomputer interfaces for communication and control", Clinical neurophysiology, 113(6), p. 767-791, 2002.

[ZAN 08] ZANDER T.O., Kothe C., WelKe S., ROetTing M., "Enhancing human-machine systems with secondary input from passive brain-computer interfaces", dans Proceedings of the 4th International BCI Workshop and Training Course, Graz, 2008.

[ZHA 17] Zhang, Z., XU, F., YAng, H., Jiang, J., CAO, Y., \& JiAO, X., "Mental fatigue detection based on the functional near infrared spectroscopy", Presented at the International Conference on Optical and Photonics Engineering (icOPEN 2016), International Society for Optics and Photonics, 2017. 